\title{
Identification and spatial distribution of glycine receptor subunits in human sperm
}

\author{
Priyadarsini Kumar and Stanley Meizel \\ Department of Cell Biology and Human Anatomy, School of Medicine, University of California, Davis, California \\ 95616, USA
}

Correspondence should be addressed to P Kumar; Email: pkumar@ucdavis.edu

\begin{abstract}
The human sperm surface glycine receptor (GLR) plays a role in an important fertilization event, the sperm acrosome reaction. Here, by western blot analysis, we report the presence of GLRA1, GLRA2, GLRA3, and GLRB subunits in human sperm. Immunolocalization studies showed that the GLRA1 and GLRA2 subunits are present in the equatorial region, the GLRA3 subunit in the flagellar principal piece, and the GLRB subunit in the acrosomal region of sperm. This first demonstration of isoforms of the sperm GLRA subunit and of a differential spatial distribution of the $\alpha$ and $\beta$ subunits on the surface of mammalian sperm suggests the possibility that human sperm GLRs have more than one function.

Reproduction (2008) 136 387-390
\end{abstract}

\section{Introduction}

The neuronal glycine receptor/ $\mathrm{Cl}^{-}$channel (GLR) is a pentameric complex consisting of three $\alpha$ and two $\beta$ subunits. Four isoforms of the $\alpha$ subunit $(\alpha 1, \alpha 2, \alpha 3$, and a4) have been identified and splice variants of the $\alpha 1$ ( $\alpha 1$ and $\alpha 1_{\text {ins }}$ ), $\alpha 2$ ( $\alpha 2 \mathrm{~A}$ and $\alpha 2 \mathrm{~B}$ ), and $\alpha 3$ ( $\alpha 3 \mathrm{~K}$ and $\alpha 3 \mathrm{~L}$ ) have been characterized (reviewed in Lynch 2004, Webb \& Lynch 2007). Recently, a splice variant of the $\beta$ subunit $(\beta \Delta 7)$ was cloned from glial cells (Oertel et al. 2007).

The acrosome is a secretory granule-like organelle in the anterior two-thirds of the sperm head, and the acrosome reaction (AR) is a modified exocytotic event that is essential for fertilization (Yanagimachi 1994, Meizel 2004). Previous pharmacological and immunochemical studies showed the involvement of a sperm GLR in the porcine, human, mouse, and hamster AR initiated by glycine and the oocyte zona pellucida or its glycoprotein ZP3 (Melendrez \& Meizel 1995, Sato et al. 2000b, Llanos et al. 2001, Bray et al. 2002, Meizel \& Son 2005). The porcine and mouse sperm studies utilized a $\mathrm{mAb}(\mathrm{mAb} 4 \mathrm{a})$ that recognizes all the subunit isoforms, while the human studies used a polyclonal antibody that recognizes both the GLRA1 and GLRA2 subunits but does not distinguish between them (Melendrez \& Meizel 1996, Sato et al. 2000a, Bray et al. 2002). Since the sperm is such a highly differentiated cell composed of a flagellum and head that are each even further compartmentalized (Yanagimachi 1994, Meizel 2004) separate localization of GLR subunits might indicate different functions. In the present study, we utilized subunit-specific antibodies to identify the GLR subunits present in human sperm and demonstrated their differential cellular localization.

\section{Results}

Western blot analysis of Triton-X-100 extracts of uncapacitated human sperm demonstrated the presence of the GLRA1 subunit that ran at an apparent molecular mass of $48.7 \pm 0.59 \mathrm{kDa}$ (Fig. 1, lane 1), the GLRA2 subunit at $49.9 \pm 1.3 \mathrm{kDa}$ (Fig. 1, lane 2), the GLRA3 subunit at $47.8 \pm 2.2 \mathrm{kDa}$ (Fig. 1, lane 3), and the GLRB subunit at $59.6 \pm 1.3 \mathrm{kDa}$ (Fig. 1, lane 4). No band was detected when the primary antibody was omitted during western blotting (Fig. 1, lane 5). These molecular masses are in agreement as previously reported for GLRA1 and GLRB subunits of the rat spinal cord GLR (Pfeiffer et al. 1982) and as predicted from the amino acid sequence for the GLRA2 and GLRA3 subunits.

Immunolocalization of the subunits in capacitated human sperm with subunit-specific antibodies showed that the GLRA1 and GLRA2 subunits are localized in the equatorial region of the sperm head (Fig. 2a and $b$ respectively), the GLRA3 subunit in the flagellar principal piece (Fig. 2d), and the GLRB subunit in the acrosomal region of the sperm head (Fig. 2e). Immunolocalization using the $\mathrm{N}$-terminal antibody that recognizes both the GLRA1 and GLRA2 subunits also showed their presence in the equatorial region (Fig. 2C). All of the localizations shown were also observed in uncapacitated sperm and were not detected when the primary 


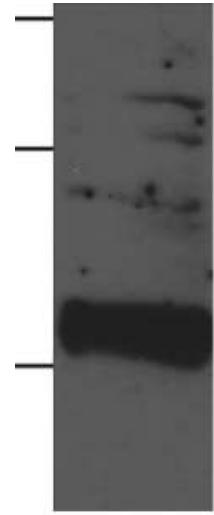

1

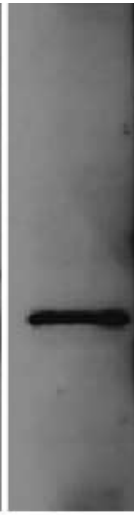

2

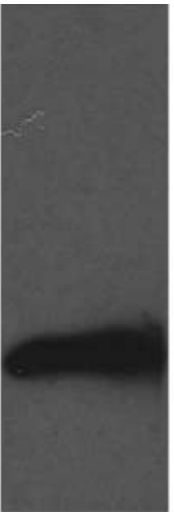

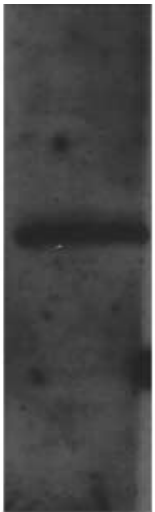

4

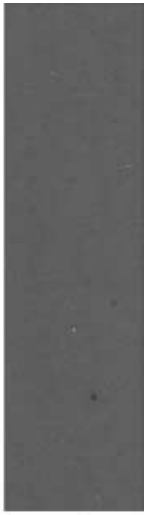

5
Figure 1 Western blot analysis of GLR subunits in human sperm: total lysates of uncapacitated human sperm equivalent to $1 \times 10^{6}$ cells were loaded onto a $10 \%$ Tris-glycine gel and blotted onto nitrocellulose membrane as described in Materials and Methods. The blots were developed with subunit specific antibodies: GLRA1 subunit (lane 1), GLRA2 subunit (lane 2), GLRA3 subunit (lane 3), GLRB subunit (lane 4 ), and only secondary antibody (lane 5). The standards in decreasing molecular weight are: phosphorylase b (97 400), serum albumin (66 200), and ovalbumin (45 000).

antibody was pre-incubated with peptide or when the primary antibody was omitted ( $n=3$, data not shown). The dim labeling seen in Fig. $2 \mathrm{a}$ is identical to that seen in controls.

\section{Discussion}

Our results are the first to demonstrate a detailed characterization (type and localization) of the different isoforms of the GLR subunits in human sperm. Earlier studies of characterization of the GLR in mouse and pig sperm used a MAB (mAb4a) antibody that recognizes all the subunits (Melendrez \& Meizel 1996, Sato et al. 2000a). In those studies, the GLR was localized on the acrosomal and equatorial regions of sperm. The GLRA1 subunit has been shown to be involved in the zona pellucida-initiated AR using mutant mice lacking the GLRA1 subunit (Meizel \& Son 2005). The sperm GLR is apparently present in the plasma membrane of the mammalian sperm head. Antibodies are not known to pass through the plasma membrane of viable sperm; yet, the GLRA1/GLRA2 antibody abolished the rhZP3initiated AR of human sperm (Bray et al. 2002) and immunolocalization of the porcine sperm GLR was identical in both fixed and viable sperm (Melendrez \& Meizel 1996).

In this study, both the GLRA1 and GLRA2 subunits were found in the equatorial region of human sperm, presumably in the plasma membrane overlying the equatorial segment, the narrowest part of the acrosome. Neither the GLRA1 nor GLRA2 subunit co-localize with the regulatory GLRB subunit that is present in the main acrosomal region (although we cannot rule out such possible co-localization in the equatorial segment of sperm). Some neuronal GLRs do exist as homomeric GLRA1 subunit receptors and the GLRA1/GLRA2 antibody has been reported to recognize only the homomeric GLRA1 receptor in such neurons (Deleuze et al. 2005). Functional homomeric neuronal GLRs consisting of $\beta$ subunits have not yet been demonstrated (Grenningloh et al. 1990). While neuronal GLRs are usually composed of both $\alpha$ and $\beta$ subunits, the lack of co-expression of the $\beta$ subunit with $\alpha$ subunit has been reported in several regions of rat brain suggesting a possibility that there could be yet another unidentified $\alpha$ subunit (Malosio et al. 1991). The GLRA4 subunit was not tested for its presence in human sperm due to the lack of the availability of a specific antibody. In addition, the human GLRA4 subunit is considered to be a pseudogene (Simon et al. 2004). Whether the human sperm head GLRB subunit exists as an unusual GLR homomer or is paired with GLRA1 and/or GLRA2 subunits in the narrow equatorial segment or is paired
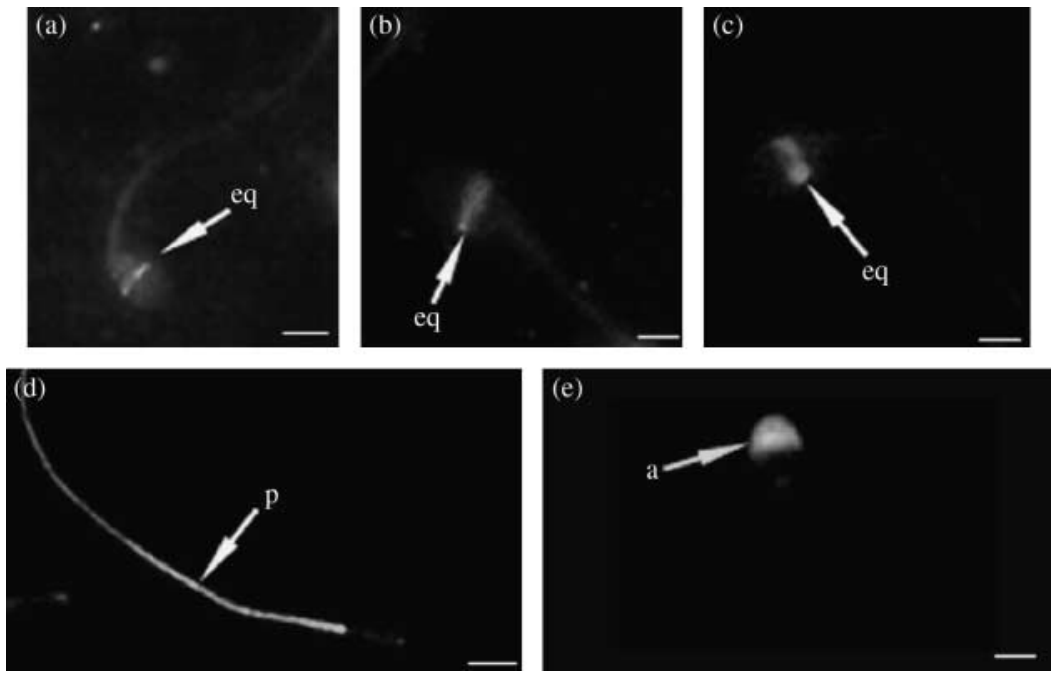

Figure 2 Immunolocalization of GLR subunits in human sperm: immunolocalization of GLR subunits was studied in capacitated sperm using (a) GLRA1 subunit, (b) GLRA2 subunit, (c) GLRA1/GLRA2 subunits, (d) GLRA3 subunit, and (e) GLRB subunit primary antibodies as described in Materials and Methods. Arrows indicate localization in the equatorial (eq), acrosomal (a), and principal-piece (p) regions of human sperm. Bar $=5 \mu \mathrm{m}$. 
with an as yet undetected $\alpha$ subunit, its spatial separation from the GLRA1 and GLRA2 subunits in the main part of the acrosome and the flagellum suggest the possibility of distinct functions for different human sperm head GLRs. For example, such diverse GLRs might be important in discrete steps during AR initiation.

Earlier studies did not detect any localization of the GLR subunits in the tail region of mice and porcine sperm. Our detection of the GLRA3 subunit in the principal piece region in the human sperm might be due to species and epitope differences. RT-PCR analysis of the GLRA3 subunit in mouse testis and sperm showed the presence of only the low molecular weight $\alpha 3 \mathrm{~K}$ splice variant (P Kumar \& S Meizel, unpublished observations). Interestingly, mice lacking the GLRA3 subunit are subfertile (the Jackson Laboratory mice database, www. jaxmice.jax.org/strain/005816.html). The presence of a GLR subunit in the flagellum suggests the possibility of a role for that receptor/channel in sperm motility. While subjective motility assays did not detect motility alterations in human sperm treated with strychnine, a GLR antagonist (Bray et al. 2002), such assays do not rule out the possibility that the sperm flagellar GLR might be involved in the hyperactivated form of sperm motility important to fertilization (Yanagimachi 1994).

In conclusion, the spatial distribution of the GLR subunits on the surface of human sperm suggests their involvement in different aspects of sperm function. Further studies will be needed to identify and elucidate such roles.

\section{Materials and Methods \\ Materials}

The following reagents were purchased: Percoll, polyvinyl alcohol from Sigma; Fraction V BSA from Serologicals Corp. (Kankakee, IL, USA); pre-cast SDS-PAGE gels, nitrocellulose membranes and broad-range molecular weight standards from Bio-Rad Laboratories; 15-ml conical polypropylene centrifuge tubes (Grenier Labortechnik) from Applied Scientific (South San Francisco, CA, USA); Donkey anti-goat horseradish peroxidase (HRP) from Jackson Immuno Research Laboratories Inc. (West Grove, PA, USA); West Pico chemiluminescence reagents from Pierce (Rockford, IL, USA); and Vectashield from Vector Laboratories (Burlingame, CA, USA). For all experiments, deionized water used was purified to $18 \mathrm{MO} \mathrm{cm}$ with a NANO-pure ion-exchange system (Barnstead/Thermolyne, Dubuque, IO, USA). All other reagents were obtained from standard sources and were of the highest purity available.

\section{Methods}

Human sperm preparation and capacitation

Protocols for human sperm studies were approved by the Human Subjects committee at the University of California, Davis, CA, USA. Semen samples were obtained by masturbation from a pool of six healthy donors. A population of $>95 \%$ motile sperm was obtained by centrifugation of semen samples through a discontinuous Percoll gradient and subsequent washing as described previously (Thomas \& Meizel 1988).

In order to undergo the AR, mammalian sperm must undergo cellular changes under defined in vitro incubation conditions or in vivo, which are collectively called capacitation (Yanagimachi 1994). Here, for immunolocalization studies, sperm were incubated under capacitating conditions (Thomas \& Meizel 1988). Washed sperm were diluted to $6 \times 10^{6} \mathrm{sperm} / \mathrm{ml}$ in a medium containing a balanced salt solution, $26 \mathrm{mg} / \mathrm{ml} \mathrm{BSA}$, $25 \mathrm{mM}$ bicarbonate, metabolites (lactate, pyruvate, and glucose), and antibiotics (penicillin and streptomycin), and incubated in $500 \mu \mathrm{l}$ aliquots in $15 \mathrm{ml}$ polypropylene centrifuge tubes for $24 \mathrm{~h}$ at $37^{\circ} \mathrm{C}$ in a $5 \% \mathrm{CO}_{2}$ /air atmosphere (Thomas \& Meizel 1988). The samples were routinely checked for capacitation by assays of their ability to undergo the progesterone-initiated AR as described earlier (Meizel \& Turner 1993).

\section{Antibodies}

The antibodies used for western blot analysis and immunolocalizations described in this paper were: goat polyclonal antibodies to human neuronal GLRA1, GLRA2, GLRA3, and GLRB subunits from Santa Cruz Biotechnology Inc. (Santa Cruz, CA, USA); rabbit polyclonal antibody to human neuronal GLRA1/GLRA2 subunits from Sigma; fluorescein isothiocyanate (FITC) goat anti-rabbit IgG from Zymed Laboratories Inc. (San Francisco, CA, USA); and FITC rabbit anti-goat IgG from Jackson ImmunoResearch Laboratories Inc. All western blots and immunolocalizations using these antibodies shown in the present report are representative of three separate experiments.

\section{Western blot analysis of the GLR subunits}

Uncapacitated human sperm were prepared by Percoll gradient centrifugation and further washing (Thomas \& Meizel 1988), and proteins were extracted using Triton-X-100 in lysis buffer $(10 \mathrm{mM}$ Tris, pH 7.4, $150 \mathrm{mM} \mathrm{NaCl}, 1 \%$ Triton-X-100 and a cocktail of protease inhibitors (1 mM EDTA, $20 \mu \mathrm{M}$ leupeptin, $1 \mu \mathrm{g} / \mathrm{ml}$ aprotinin, $1 \mathrm{mM}$ 4-(2-aminoethyl) benzenesulfonyl fluoride hydrochloride, $1 \mathrm{mM}$ benzamidine $\mathrm{HCl}$, $1 \mu \mathrm{M}$ pepstatin $\mathrm{A}$, and $1 \mu \mathrm{M} \mathrm{E}-64))$. The extract was spun at $10000 \mathrm{~g}$ for $20 \mathrm{~min}$ and the supernatant was run on a $10 \%$ SDS-PAGE gel and the transferred to nitrocellulose membrane at $100 \mathrm{~mA}$ constant current for $2 \mathrm{~h}$. The membrane was blocked for $2 \mathrm{~h}$ with $3 \% \mathrm{BSA}(\mathrm{w} / \mathrm{v})$ in TBST $(10 \mathrm{mM}$ Tris $(\mathrm{pH}$ 7.4), $150 \mathrm{mM} \mathrm{NaCl}, 0.05 \%$ Tween 20) and then incubated overnight at $4{ }^{\circ} \mathrm{C}$ with a $1: 5000$ dilution of primary antibody diluted in $1 \%$ BSA $(\mathrm{w} / \mathrm{v})$ in TBST. The membrane was washed three times with TBST and incubated with a 1:25 000 dilution of donkey anti-goat antibody conjugated to HRP for $1 \mathrm{~h}$ at room temperature. The membrane was subsequently washed extensively with TBST and developed by chemiluminescence.

\section{Immunolocalization of GLR subunits}

For immunolocalization experiments, uncapacitated or capacitated human sperm (75-85\% motile) were fixed with $2 \%$ paraformaldehyde for $10 \mathrm{~min}$ at room temperature. The cells were then washed three times with PBS $(\mathrm{pH}$ 7.4) and plated on 
poly-L-lysine coated slides. The cells were allowed to adhere for 15 min and washed twice with PBS and washed four times for 5 min each with PBS. In the case of the $\alpha 1$ antibody, the cells were permeabilized with $0.1 \%$ Triton-X-100 for 5 min before washing with PBS. The slides were then blocked with $0.2 \%$ gelatin for $2 \mathrm{~h}$ and incubated overnight at $4{ }^{\circ} \mathrm{C}$ with primary antibody $(100 \mu \mathrm{g} / \mathrm{ml}$ antibody). The slides were then washed with PBS and incubated for $1 \mathrm{~h}$ with $10 \mu \mathrm{g} / \mathrm{ml}$ of the respective secondary antibodies and were washed as described above. The slide was mounted with Vectashield and sperm was examined using a Nikon E800 epifluorescence microscope at $1000 \times$ magnification.

\section{Declaration of interest}

The authors declare that there is no conflict of interest that could be perceived as prejudicing the impartiality of the research reported.

\section{Funding}

This work was supported by NIH grant HD 33368 to S M.

\section{References}

Bray C, Son JH, Kumar P, Harris JD \& Meizel S 2002 A role for the human sperm glycine receptor/Cl(-) channel in the acrosome reaction initiated by recombinant ZP3. Biology of Reproduction 66 91-97.

Deleuze C, Runquist M, Orcel H, Rabie A, Dayanithi G, Alonso G \& Hussy N 2005 Structural difference between heteromeric somatic and homomeric axonal glycine receptors in the hypothalamo-neurohypophysial system. Neuroscience 135 475-483.

Grenningloh G, Pribilla I, Prior P, Multhaup G, Beyreuther K, Taleb O \& Betz H 1990 Cloning and expression of the $58 \mathrm{kDa}$ beta subunit of the inhibitory glycine receptor. Neuron 4 963-970.

Llanos MN, Ronco AM, Aguirre MC \& Meizel S 2001 Hamster sperm glycine receptor: evidence for its presence and involvement in the acrosome reaction. Molecular Reproduction and Development 58 205-215.

Lynch JW 2004 Molecular structure and function of the glycine receptor chloride channel. Physiological Reviews 84 1051-1095.

Malosio ML, Marqueze-Pouey B, Kuhse J \& Betz H 1991 Widespread expression of glycine receptor subunit mRNAs in the adult and developing rat brain. EMBO Journal 10 2401-2409.
Meizel S 2004 The sperm, a neuron with a tail: 'neuronal' receptors in mammalian sperm. Biological Reviews of the Cambridge Philosophical Society $79713-732$.

Meizel S \& Son JH 2005 Studies of sperm from mutant mice suggesting that two neurotransmitter receptors are important to the zona pellucidainitiated acrosome reaction. Molecular Reproduction and Development 72 250-258.

Meizel S \& Turner KO 1993 Effects of polyamine biosynthesis inhibitors on the progesterone-initiated increase in intracellular free $\mathrm{Ca}^{2+}$ and acrosome reactions in human sperm. Molecular Reproduction and Development 34 457-465.

Melendrez CS \& Meizel S 1995 Studies of porcine and human sperm suggesting a role for a sperm glycine receptor/Cl-channel in the zona pellucida-initiated acrosome reaction. Biology of Reproduction $\mathbf{5 3}$ 676-683.

Melendrez CS \& Meizel S 1996 Immunochemical identification of the glycine receptor/Cl-channel in porcine sperm. Biochemical and Biophysical Research Communications 223 675-678.

Oertel J, Villmann C, Kettenmann H, Kirchhoff F \& Becker CM 2007 A novel glycine receptor beta subunit splice variant predicts an unorthodox transmembrane topology. Assembly into heteromeric receptor complexes. Journal of Biological Chemistry 282 2798-2807.

Pfeiffer F, Graham D \& Betz H 1982 Purification by affinity chromatography of the glycine receptor of rat spinal cord. Journal of Biological Chemistry 257 9389-9393.

Sato Y, Son JH \& Meizel S 2000a The mouse sperm glycine receptor/chloride channel: cellular localization and involvement in the acrosome reaction initiated by glycine. Journal of Andrology 21 99-106.

Sato Y, Son JH, Tucker RP \& Meizel S 2000b The zona pellucida-initiated acrosome reaction: defect due to mutations in the sperm glycine receptor/Cl(-) channel. Developmental Biology 227 211-218.

Simon J, Wakimoto H, Fujita N, Lalande M \& Barnard EA 2004 Analysis of the set of $\mathrm{GABA}(\mathrm{A})$ receptor genes in the human genome. Journal of Biological Chemistry 279 41422-41435.

Thomas P \& Meizel S 1988 An influx of extracellular calcium is required for initiation of the human sperm acrosome reaction induced by human follicular fluid. Gamete Research 20 397-411.

Webb TI \& Lynch JW 2007 Molecular pharmacology of the glycine receptor chloride channel. Current Pharmaceutical Design 13 2350-2367.

Yanagimachi R 1994 Mammalian fertilization. In The Physiology of Reproduction, pp 189-317. Eds E Knobil \& JD Neill. New York: Raven Press.

Received 22 May 2008

First decision 12 June 2008

Accepted 8 July 2008 